\title{
Protective roles of ketamine and xylazine against light- induced retinal degeneration in rats
}

\author{
Xuefeng Li, Tao Li, Li Yang, Long-Yun Li* \\ Department of Anesthesiology, China-Japan Union Hospital of Jilin University, Changchun, Jilin130033, China \\ *For correspondence: Email: BarrygPerezhv@yahoo.com; Tel: 0086-0431-84997222
}

Sent for review: 23 July 2018

Revised accepted: 18 March 2019

\begin{abstract}
Purpose: To study the protective effects of ketamine and xylazine against light exposure-induced retinal degeneration $(R D)$ in rats.

Methods: Sprague Dawley rats were divided into three groups viz: light damage before anesthesia (LAE), light damage only (LDO), and control (CON) group which was kept in the dark for $12-18 \mathrm{~h}$ to habituate before light exposure. LDO group was exposed to light before anesthesia, while LAE group was maintained under anesthesia with ketamine and xylazine. The groups were kept for $120 \mathrm{~min}$ in darkness after anesthesia prior to light exposure and they were awakened prior to light damage. Functional assessment was carried out using electroretinography while morphological analysis was carried out using histology and immunochemistry techniques.

Results: Ketamine-xylazine combination preserved the function of the retina and protected against light-induced $R D$ based on retinal imaging studies and immunochemistry analysis. Xylazine and ketamine anesthesia provided protection against light-induced retinal damage, and thus reduced photoreceptor cell death.

Conclusion: These results indicate that xylazine and ketamine anesthesia offer protection against lightinduced damage and photoreceptor cell death in rats, and therefore, can potentially be developed for use in humans.
\end{abstract}

Keywords: Retinal degeneration, Light damage, Protection, Xylazine, Ketamine

\begin{abstract}
This is an Open Access article that uses a fund-ing model which does not charge readers or their institutions for access and distributed under the terms of the Creative Commons Attribution License (http://creativecommons.org/licenses/by/4.0) and the Budapest Open Access Initiative (http://www.budapestopenaccessinitiative.org/read), which permit unrestricted use, distribution, and reproduction in any medium, provided the original work is properly credited.

Tropical Journal of Pharmaceutical Research is indexed by Science Citation Index (SciSearch), Scopus, International Pharmaceutical Abstract, Chemical Abstracts, Embase, Index Copernicus, EBSCO, African Index Medicus, JournalSeek, Journal Citation Reports/Science Edition, Directory of Open Access Journals (DOAJ), African Journal Online, Bioline International, Open-J-Gate and Pharmacy Abstracts
\end{abstract}

\section{INTRODUCTION}

Retinal degeneration (RD) is a condition of retinopathy which leads to retinal degeneration due to death of the retinal cells as a result of loss of light-sensitive photoreceptors [1]. Photoreceptor cells are involved in the visual process. Thus, their death results ultimately in RD. In the current investigation, retinal light damage in rats was used as a model for studies on the prevention of retinal degeneration in humans using neuroprotective approaches [2]. Neuroprotection involves the preservation and protection of the structure and function of neuronal cells after an acute insult through a mechanism involving minimization of damage to these cells, and maximization of their recovery [3].

Sometimes, neuroprotection is a prophylactic 
measure at the peri-ischemic period in order to enhance the survival of neuronal cells. Indeed, substantial research attention has been focused on identifying agents/chemicals agents that reduce neuronal injury during anesthesia. There are also studies on the effect of anesthetics on the physiopathology of ischemia in neuronal damage in animal models [4]. The main goal of neuroprotective treatments is to reduce cerebral damage, thereby increasing the tolerance of neurological tissue to ischemia and changing the intracellular responses to energy supply deprivation. Moreover, there are reports on the neuroprotective effect of anesthetics against ischemic insults and other neurodegenerative diseases including Parkinson disease (PD) and Alzheimer disease (AD) in rats.

The neuroprotective effects of chemical agents such as chloral hydrate-carprofen, ketaminexylazine and fentanyl-medetomidine for anesthesia has been reported in in vivo studies in the retinal cells of various animal models [5]. Light has the ability to damage the retina (phototoxicity), thereby decreasing the responses of the photoreceptor to light. Among the different components of visible light, the blue colour between the wavelengths of 400 and $500 \mathrm{~nm}$ is considered the most harmful component. Several experiments have demonstrated the use of animal models to investigate RD caused by light damage which may occur due to external factors or diseases [6].

Studies have also shown that light-induced RD model in gas inhaled anesthesia leads to neuroprotection against photoreceptor apoptosis. The present study was aimed at investigating the protective effect of ketamine and xylazine against light-induced retinal degeneration in rats. The induction of $\mathrm{RD}$ was based on the protocol developed by Arango-Gonzalez et al [7]. The neuro-protective use of ketamine-xylazine on RD has been reported in various researches.

The present investigation focused on its potential protective effect against RD.

\section{EXPERIMENTAL}

\section{Ethical statement}

The study protocol was approved by Jilin University Ethical Committee (approval no. JUEC/ANE/12252017). All procedures were performed in accordance with the guidelines of National Institute of Health for Care and Use of Laboratory Animals [8].

\section{Experimental animals}

The animals were handled as per the National Institute of Health, USA Guidelines for Care and Use of Laboratory Animals [8]. Thirty SpragueDawley rats, weighing 290 - $350 \mathrm{~g}$ were used in the study. The rats were housed in polypropylene cages with standard laboratory conditions at an optimum room temperature of $22{ }^{\circ} \mathrm{C}$, with a $12 \mathrm{~h}$ light/12 $\mathrm{h}$ dark cycle and 200 lux of illumination.

\section{Anesthesia and light exposure}

The rats were divided into three groups viz. LDO, LAE, and CON (Table 1). They were kept in the dark for $12-18 \mathrm{~h}$ to habituate before the light exposure. Initially, the LDO group was exposed to light before anesthesia, while the LAE group was maintained under anesthesia by injecting with ketamine $(80 \mathrm{mg} / \mathrm{kg} 0$ and xylazine $(10 \mathrm{mg} / \mathrm{kg}$ ) for at least $90 \mathrm{~min}$. These groups were kept for another 120 min in darkness after anesthesia prior to light exposure. The rats were awakened prior to light-induced damage, and their pupils were dilated $40 \mathrm{~min}$ with tropicamide (one drop to each pupil) to allow for light damage. To prevent closure of the eyes, the rats were monitored every 15 - $20 \mathrm{~min}$. Then, the LDO and LAE groups were exposed for $120 \mathrm{~min}$ to light with a brightness of 14,000 lux, after which they were transferred to darkness for about $14 \mathrm{~h}$ as per the standard protocol [9]. The rats in the control group were not exposed to anesthesia and light illumination.

\section{Electroretinography (ERG)}

Before the exposure to light, the baseline electroretinography (ERG) of the LDO and LAE rat groups were recorded every 10 days. In addition, the baseline ERG recording was carried out once every 10 days after the light-induced damage. The rats were adapted to $12 \mathrm{~h}$ of darkness and one drop of tropicamide was applied to the pupils. After $30 \mathrm{~min}$, the measurement of ERG was carried out using an active electrode. To avoid keratopathy, methylcellulose was added. The electrode was introduced between the eyes and the tail to act as the reference. The ERG recording protocol comprised various steps for measuring the strength of the stimuli produced by white light using ColorDomeH Simulator. The flash duration was set at $5 \mathrm{~ms}$ and the scotopic flashes were delivered without any background illumination. ERG data analysis was carried out using Delphi 7.0. The oscillatory data were extracted using discrete Fourier transform $(80-290 \mathrm{~Hz})$, and the a-wave and b-waves were analyzed. However, for light flashes with low intensity, the rod 
sensitivity was estimated by fitting the NakaRushton fit [10]. Furthermore, the a-wave and bwave were compared with those of the control groups.

\section{In vivo retinal imaging}

The LDO and LAE groups were exposed to light, and retinal imaging was conducted on the 2 nd day and on the 10th day. The control groups were kept for reference. Based on the protocol described by Zhang et al, confocal scanning laser ophthalmoscopy (cSLO) and spectral domain optical coherence tomography (SDOCT) imaging were conducted [11]. The imaging of the central region of peripapillary was done with the near-infrared channel, while the horizontal and vertical SD-OCT sections were recorded perpendicularly. Eye Explorer 1.6 was employed for quantifying the retinal thickness based on the vertical scan. The total retinal thickness (TRT) and outer nuclear layer thickness (ONL) of the LAE, LDO, and control groups were quantified on the 8th loci towards the periphery. Furthermore, the segmentation line detected the TRT.

\section{Histology and immunohistochemical analysis}

The rats were sacrificed without any physical pain and the lenses and the anterior parts were excised from the eyes. They were further treated with $4 \%$ PFA in $0.1 \mathrm{M} \mathrm{PO}_{4}$ at $4{ }^{\circ} \mathrm{C}$ for $60 \mathrm{~min}$ and dehydrated in ethanol. They were then embedded in paraffin after immersing in chloroform. Paraffin sections were radically sliced at $5 \mathrm{~mm}$ and kept at $4^{\circ} \mathrm{C}$. The $\mathrm{ONL}$, thickness and the segments were quantified and at equal distances. For immunohistochemical studies, the paraffin sections were washed and rehydrated. The antigen was retrieved by treating with $0.1 \mathrm{M}$ citrate buffer, $\mathrm{pH} 5.8$ under pressure for $12 \mathrm{~min}$. This was followed by cooling the section with the specific antibodies. The radial sections were further treated with PBS $(70 \mathrm{mM}$, $\mathrm{pH}$ 7.2) consisting of $0.05 \%$ Triton $\mathrm{X}-100$ and goat serum (15\%) for $120 \mathrm{~min}$. The sections were then incubated at $4{ }^{\circ} \mathrm{C}$ with their corresponding antibodies for $24 \mathrm{~h}$. The reactions were observed with a standard anti-rabbit antibody (Alexa Fluor 488) diluted in the ratio of 1: 600 . The sections from the controls were processed without anti-rabbit antibody. Photomicrographs were recorded for the superior region of the retina using a microscope.
In each group, data were obtained from three different rats chosen randomly. The data analysis also includes measurement of the control rats for evocative assessment. For the quantification of the captured cells, snapshots of whole radial slices were magnified and the cells were manually counted. The total cells were calculated by the dividing the ONL with average cell size, and with total ONAL to obtain the percentage value

\section{Statistical analysis}

Statistical analysis was performed using SPSS 18.0 (SPSS Inc, USA). The ERG parameters were analysed based on the ratio between baseline data and follow-up period. Data analysis for histochemical studies was carried out using $t$ test.

\section{RESULTS}

There was no statistically significant difference in the ERG response between the retinas of the LDO and LAE groups in the baseline observations and measurements. Figure 1 shows the ERG curves of the LAE and LDO groups based on the function of scotopic sensitivity. The results show a positive influence on the function of rod on-bipolar cells and on Muller cells. The analysis of variance also revealed that the rod cells of the retina were reduced in quantity, but their function was not affected. Figure 2 depicts the comparison of the a-wave amplitudes of LDO and LAE groups based on the strength of the stimulation. It is observed in Figure 2 that the awaves showed higher amplitudes in the case of LAE group, when compared to the LDO group, due probably to scotopic conditions. These might be due the functions of retinal rods and cones which are photoreceptors for low light levels. There was not much difference in the a-wave implicit times in the LAE group, an indication of its photoreceptor protection capability. However, the gradient lines of a-wave were high for the LAE group on the 13th day after the exposure to light. The a-wave is an indicator which is electrophysiological in nature and it represents the process of photo-transduction in the sensory neurons of the retina. Thus, photo-transduction and the photoreceptor cells of the retina were preserved in this group.

Table 1: Rat groups used in the study

\begin{tabular}{lcc}
\hline Group & Light exposure & Ketamine-xylazine treatment \\
\hline Light damage before anesthesia (LAE) & Positive & Positive \\
Only light damage (LDO) & Positive & Negative \\
Control (CON) & Negative & Negative \\
\hline
\end{tabular}


Figure $3 \mathrm{~A}$ shows that the amplitude of $\mathrm{b}$-wave ratio for the LAE group had a high-intensity stimulus, when compared to the LDO group, as evidenced from Figure 3B.

The in vivo retinal analysis on TRT and ONL during the $2^{\text {nd }}$ and $10^{\text {th }}$ days is depicted in Figure 4 ( $A$ and $C$ ). The graph shows that there was no significant difference in the retinal layering, when compared to the control rats. On the other hand, rats in the LDO group showed a significant difference in ONL thickness, when compared to rats in the LAE group. However, TRT thickness was comparable between the two groups. Ten days after the light damage (Figure 4B and D),

TRT and ONL thickness were reduced in the LDO group, when compared to the LAE group. Thus, the in vivo retinal analysis suggests that the formation of retinal edema on the 2 nd day after the light-induced damage, as well as the reduction in the ONL thickness may cause the death of photoreceptor cells.

Figure 5 depicts retinal section of the lightinduced damage after $36 \mathrm{~h}$. There was no significant difference in GFAP staining between the LAE and LDO groups, as depicted Figure 6. Moreover, rhodopsin stain revealed that there was not much difference in immune-labelling between the two groups (Figure 7). The rhodopsin stain also depicted a normal distribution which was restricted to the photoreceptor OS.
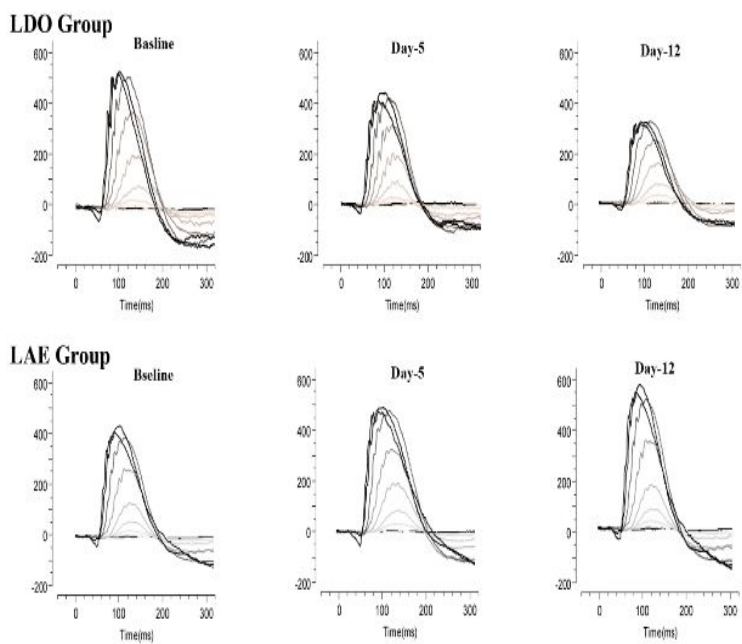

Figure 1: Responses of ERG curves to nine flashes recorded at baseline, day 5 and day 12
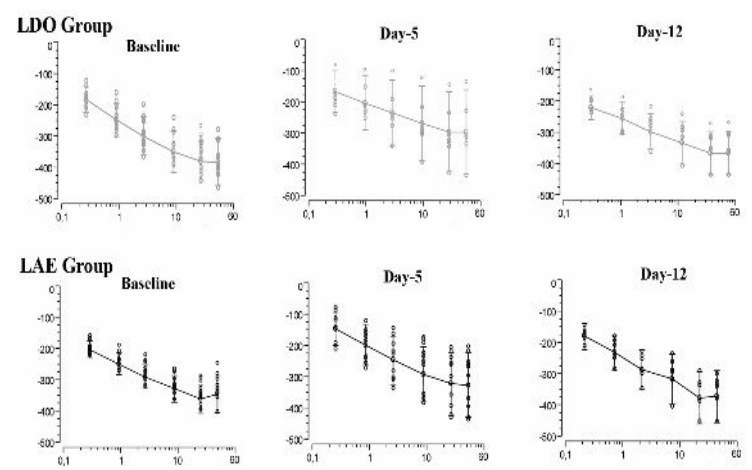

Figure 2: Comparison of amplitudes of a-wave between LDO and LAE groups
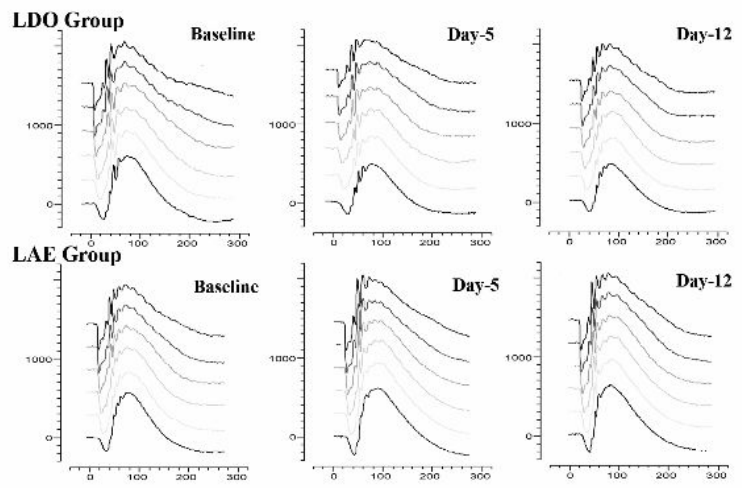

Figure 3: Comparison of amplitudes of b-wave between LDO and LAE groups

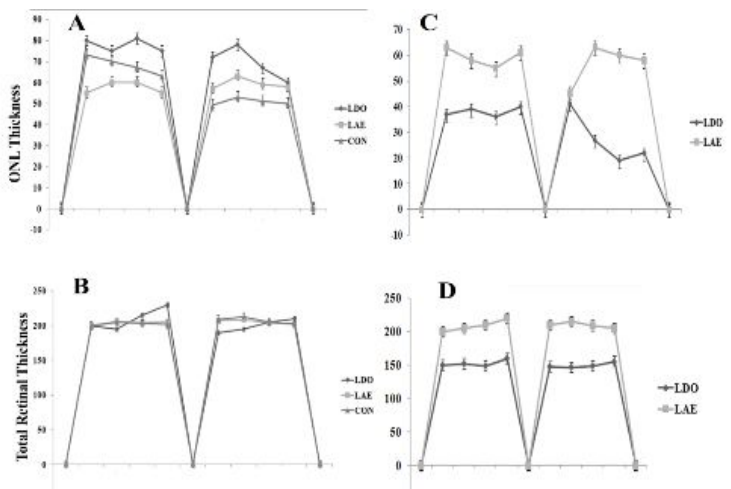

Figure 4: In vivo analysis of LDO, LAE and control groups based on total retinal thickness
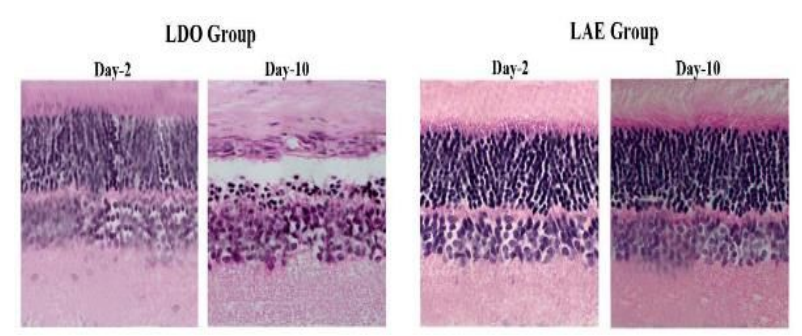

Figure 5: Hematoxylin and eosin (H\&E) staining of retinal sections from LDO and LAE groups 


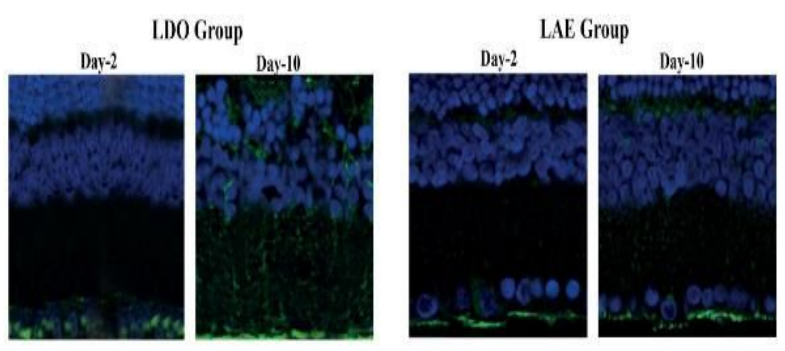

Figure 6: GFAP staining of retinal sections from LDO and LAE groups
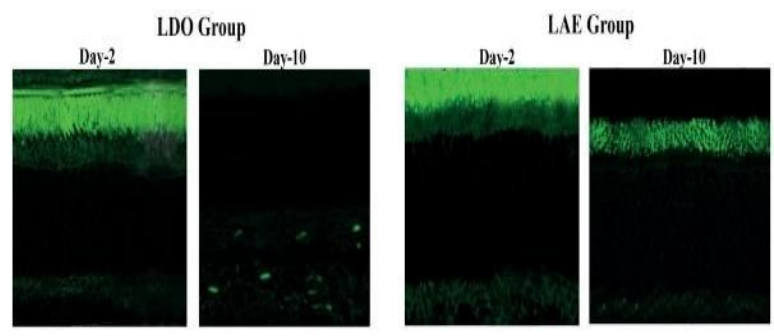

Figure 7: Rhodopsin staining of retinal sections from LDO and LAE groups

\section{DISCUSSION}

This study investigated the protective effect of ketamine and xylazine on light-induced retinal damage. Previous studies have been carried out on potential protective effects of anesthesia against retinal degeneration. Some studies have proposed mechanisms involved in the positive outcome of ketamine-xylazine anesthesia [12]. There are also reports which indicate the role of anesthetic agents as neuroprotective drugs [13]. On the other hand, various workers have reported contrasting results from studies on the effect of anesthetics on light-induced RD, thereby making complicated the understanding of the various findings [14]. Moreover, these investigations used different procedures and parameters to determine RD [15]. In addition, the tools and techniques used in various anesthetic procedures for light damage induction are different [16].

However, all the studies reported on the neuroprotective effect against light-induced RD in various animal models. Keller et al reported that halothane anesthesia reversibly inhibited metabolic regeneration of rhodopsin, thereby preventing further absorption of photons during the exposure of light [17]. In their study, Keller et al. found that halothane anesthesia prevented retinal degeneration induced by white light, but not that induced by blue light. Their study further observed that the halothane-anesthetized photoreceptors of mice and rats were protected from white light-induced RD but not from bluelight-induced damage to retinal cells [17]. This is because halothane cannot protect the retina from blue light which has the capability of restoring the function of rhodopsin from photo-reversal bleaching. This process usually allows rhodopsin molecules to capture light molecules required for the induction of RD [18].

Rhodopsin is a light-sensitive receptor protein which main function is transduction of visual signals. It is usually present in the rods of the retina which are very photo-sensitive, and constitute the major factor involved in visibility in low-intensity light environments [19]. Mutation of rhodopsin gene results in various retinopathies. In many cases, the protein arising from the mutation accumulates with ubiquitin thereby damaging the whole network cycle. In some cases, the cells are unable to degrade this mutated non-functional protein, resulting in apoptosis of the photoreceptor cells [20].

In addition, Grimm et al observed that exposure of rats to blue light rats caused severe retinal damage and activation AP-1 transcription factor [21]. It was also observed that green light exerted no severe effect [21]. However, in the present study, exposure of light quickly resulted in RD which can be compared to damage after mild exposure to blue light. It was also observed that the dosage of ketamine-xylazine used was sufficient to prevent the light damage. A similar effect was also observed by Keller et al who used halothane anesthesia [17]. This may be because ketamine alone has several limitations and it is known for its weak muscle relaxant properties. It is mainly used with a mixture of other anesthetics e.g. diazepam or xylazine [22]. On the other hand, xylazine is an agonist of the a2 class of adrenergic receptors. It produces sedation and muscle relaxation in various nonhuman mammals such as horse, cattle and cats. In the absence of xylazine, the neuroprotective effects of ketamine are still observed in some experiments, which suggests that xylazine has nothing to do with the neuroprotective effect. Thus, ketamine plays important role in the neuroprotection of RD [23].

The main objective of the present study was to determine the positive outcome of the use of ketamine-xylazine as anesthesia, and its role in neuroprotection in a light-induced RD model. An obvious neuroprotective effect of ketaminexylazine was observed. These findings also provide insights into the existing reports on the implications of light damage for inducing RD in various animal models.

On the other hand, there are also reports which show that rhodopsin regeneration in rats is 
slowed down by ketamine-xylazine combination [15]. It has been reported that ketamine-xylazine combination protects against light-induced damage caused by the activation of NOS. Moreover, ketamine preserves the morphology of the neuronal cells by increasing their viability [24]. This is because ketamine downregulates the expression of proteins that control the transcription of DNA such as nuclear factor kappa-light-chain-enhancer of activated B cells (NF-kB). In many cases of neurodegenerative orders (including retinal degeneration), NF-KB is activated [25].

The present study observed a specific position in both the LAE and LDO rats in their retina. These regions may be responsible for the light-induced damage and thinning of the retinal cells. The molecular process involved in this particular damage in this localized region is not clearly understood. It may be caused by the higher level of rhodopsin.

\section{Study limitations}

The population size of the rats used in this investigation is too small for statistical and morphological analyses. Moreover, the biological relevance of neuroprotection requires further studies to unravel the biochemical mechanisms involved.

\section{CONCLUSION}

The study demonstrates that xylazine and ketamine anesthesia provide protection against light-induced retinal damage, and reduces the death of photoreceptors in rats. Thus, the use of the anesthetic mixture has clinical potentials for its neuroprotective effect in humans.

\section{DECLARATIONS}

\section{Acknowledgement}

The authors acknowledge the support of ChinaJapan Union Hospital of Jilin University, Changchun, Jilin, China. This study was financed by China-Japan Union Hospital of Jilin University, Changchun, Jilin, China.

\section{Conflict of interest}

No conflict of interest is associated with this work. The funding organization(s) played no role in the study design nor in the collection, analysis, and interpretation of data; writing of the report or in the decision to submit the report for publication.

\section{Contribution of Authors}

The authors declare that this work was done by the authors named in this article and all liabilities pertaining to claims relating to the content of this article will be borne by them.

\section{Open Access}

This is an Open Access article that uses a funding model which does not charge readers or their institutions for access and distributed under the terms of the Creative Commons Attribution License (http://creativecommons.org/licenses/by/ 4.0) and the Budapest Open Access Initiative (http://www.budapestopenaccessinitiative.org/rea d), which permit unrestricted use, distribution, and reproduction in any medium, provided the original work is properly credited.

\section{REFERENCES}

1. Sullivan LS, Daiger SP. Inherited retinal degeneration: exceptional genetic and clinical heterogeneity. Mol Med Today1996; 2(9): 380-386.

2. Lee ES, Flannery JG. Transport of Truncated Rhodopsin and Its Effects on Rod Function and Degeneration. Invest. Ophthalmol. Vis Sci 2007; 48(6): 2868-2876.

3. An MJ, Kim CH, Nam GY, Kim DH, Rhee S, Cho SJ, Kim JW. Transcriptome analysis for UVB-induced phototoxicity in mouse retina. Environ Toxicol2018; 33(1):52-62.

4. Wang $Y, B i X$, Baudry M. Calpain-2 as a therapeutic target for acute neuronal injury. Expert Opin Ther Targets2018; 22(1): 19-29.

5. Maetzler W, Liepelt I, Berg D. Progression of Parkinson's disease in the clinical phase: potential markers. Lancet Neurol2009; 8(12): 1158-1171.

6. Kawaguchi M, Furuya $H$, Patel PM. Neuroprotective effects of anesthetic agents. J Anesth 2005; 19(2):150156.

7. Hansen KR, DeWalt GJ, Mohammed Al, Tseng $H A$, Abdulkerim ME, Bensussen S, Saligrama V, Nazer B, Eldred WD, Han X. Mild Blast Injury Produces Acute Changes in Basal Intracellular Calcium Levels and Activity Patterns in Mouse Hippocampal Neurons. J Neurotrauma 2018; doi: 10.1089/neu.2017.5029.

8. Guide for the Care and Use of Laboratory Animals: 8 Edn. https://www.nap.edu/read/12910/chapter/1. Accessed 2 April 2019.

9. Schifilliti D, Grasso G, Conti A, Fodale V. Anaestheticrelated neuroprotection: intravenous or inhalational agents? CNS Drugs 2010; 24: 893-907.

10. Ferro MM, Angelucci MEM, Anselmo-Franci JA, Canteras NS, Da Cunha C. Neuroprotective effect of ketamine/xylazine on two rat models of Parkinson's disease. Braz J Med Biol Res 2007; 40: 89-96. 
11. Organisciak DT, Vaughan DK. Retinal light damage: mechanisms and protection. Prog. Retin Eye Res2010;29: 113-134.

12. Vicente-Tejedor J, Marchena M, Ramırez L, GarcıaAyuso D, Gomez-Vicente V, SanchezRamos C. Removal of the blue component of light significantly decreases retinal damage after high intensity exposure. PLOS ONE 2018;13(3): e0194218.

13. Woicke J, Haile S, Mysore J, Peden WM, Lejeune T, Sanderson T, Brodie T. Spontaneous Findings in the Eyes of Cynomolgus Monkeys (Macaca fascicularis) of Mauritian Origin. Toxicol Pathol2018: doi: 10.1177/ 0192623318758619.

14. Chen X, Hall H, Simpson JP, Leon-Salas WD, Ready DF, Weake VM. Cytochrome b5 protects photoreceptors from light stress-induced lipid peroxidation and retinal degeneration. NPJ Aging Mech Dis2017; 3:18-25

15. Arango-Gonzalez B, Schatz A, Bolz S, EslavaSchmalbach J, Willmann G. Effects of Combined Ketamine/Xylazine Anesthesia on Light Induced Retinal Degeneration in Rats. PLOS ONE 2012;7(4): e35687.

16. Cubizolle A, Guillou L, Mollereau B, Hamel CP, Brabet $P$. Fatty acid transport protein 1 regulates retinoid metabolism and photoreceptor development in mouse retina. PLoS One 2017; 12(7): e0180148.

17. Joshi NR, Ly E, Viswanathan S. Intensity response function of the photopic negative response (PhNR): effect of age and test-retest reliability. Doc Ophthalmol 2017; 135(1):1-16

18. Zhang P, Mocci J, Wahl DJ, Meleppat RK, Manna SK, Quintavalla M, Muradore R, Sarunic MV, Bonora S, Pugh EN Jr., Zawadzki RJ. Effect of a contact lens for in vivo mouse retinal imaging: Effective focal length changes and monochromatic aberrations. Exp Eye Res 2018;172: 86-93.

19. Tosini G, Ferguson I, Tsubota K. Effects of blue light on the circadian system and eye physiology. Mol Vis2016, 22: 61-72.

20. Marquioni-Ramella MD, Suburo AM. Photo-damage, photo-protection and age-related macular degeneration. Photochem. Photobiol Sci2015; 14(9): 1560-1577.

21. Hüske C, Sander SE, Hamann, Kershaw O, Richter F, Richter A. Towards optimized anesthesia protocols for stereotactic surgery in rats: Analgesic, stress and general health effects of injectable anesthetics. A comparison of a recommended complete reversal anesthesia with traditional chloral hydrate monoanesthesia. Brain Res 2016; 1642: 364-375.
22. Kolesnikov AV, Maeda A, Tang PH, Imanishi $Y$, Palczewski K, Kefalov VJ. Retinol dehydrogenase 8 and ATP-binding cassette transporter 4 modulate dark adaptation of $M$-cones in mammalian retina. $J$ Physiol2015;593(22): 4923-4941.

23. Baluška F, Yokawa K, Mancuso S, Baverstock K. Understanding of anesthesia - Why consciousness is essential for life and not based on genes. Commun Integr Biol2016; 9(6): e1238118.

24. Keller C, Grimm C, Wenzel A, Hafezi F, Reme C. Protective effect of halothane anesthesia on retinal light damage: inhibition of metabolic rhodopsin regeneration. Invest Ophthalmol Vis Sci2001;42: 476-480.

25. Grimm C, Remé CE, Rol PO. Williams TP. Blue light's effects on rhodopsin: photoreversal of bleaching in living rat eyes. Invest. Ophthalmol Vis Sci 2000;41(12): 39843990.

26. Nakamura Y, Yasuike M, Mekuchi M, Iwasaki Y, Ojima N, Fujiwara A, Chow S, Saitoh K. Rhodopsin gene copies in Japanese eel in a teleost-specific genome duplication. Zoological Lett 2017; 3: 18

27. Saliba RS, Munro PM, Luthert PJ, Cheetham ME. The cellular fate of mutant rhodopsin: quality control, degradation and aggresome formation. J Cell Sci2002; 15(Pt 14): 2907-29018.

28. Grimm C, Wenzel A, Williams T, Rol P, Hafezi $F$ (2001) Rhodopsin-mediated blue-light damage to the rat retina: effect of photoreversal of bleaching. Invest. Ophthalmol Vis Sci 42: 497-505.

29. Lissek T, Obenhaus HA, Ditzel DA, Nagai T, Miyawaki A, Sprengel R, Hasan MT. General Anesthetic Conditions Induce Network Synchrony and Disrupt Sensory Processing in the Cortex. Front Cell Neurosci2016; 10: 64.

30. Rozza A, Masoero E, Favalli L, Lanza E, Govoni S. Influence of different anaesthetics on extracellular amino acids in rat brain. J Neurosci Methods 2000; 101: 165169.

31. Hau S, Reich DM, Scholz M, Naumann W, Emmrich F, Kamprad M, Boltze J. Evidence for neuroprotective properties of human umbilical cord blood cells after neuronal hypoxia in vitro. BMC Neurosci 2008; 9: 30.

32. Sakamoto K1, Okuwaki T, Ushikubo H, Mori A, Nakahara $T$, Ishii K. Activation inhibitors of nuclear factor kappa $B$ protect neurons against the NMDA-induced damage in the rat retina. J Pharmacol Sci2017; S1347-8613(17): 30162-30167. 